(1)

CrossMark

\title{
Aminopeptidase N/CD13 as a potential therapeutic target in malignant pleural mesothelioma
}

\author{
Takahiko Otsuki ${ }^{1}$, Taku Nakashima ${ }^{1}$, Hironobu Hamada ${ }^{2}$, Yusuke Takayama $^{3}$, \\ Shin Akita ${ }^{1,4}$, Takeshi Masuda ${ }^{1}$, Yasushi Horimasu ${ }^{1}$, Shintaro Miyamoto', \\ Hiroshi Iwamoto ${ }^{1}$, Kazunori Fujitaka (10) ${ }^{1}$, Yoshihiro Miyata ${ }^{5}$, Masayuki Miyake ${ }^{6}$, \\ Nobuoki Kohno ${ }^{7}$, Morihito Okada ${ }^{5}$ and Noboru Hattori ${ }^{1}$
}

Affiliations: ${ }^{1}$ Dept of Molecular and Internal Medicine, Graduate School of Biomedical and Health Sciences, Hiroshima University, Hiroshima, Japan. ${ }^{2}$ Dept of Physical Analysis and Therapeutic Sciences, Graduate School of Biomedical and Health Sciences, Hiroshima University, Hiroshima, Japan. ${ }^{3}$ Dept of Hematology and Respiratory Medicine, Kochi University, Kochi, Japan. “Dept of Respiratory Medicine, Chugoku Rosai Hospital, Hiroshima, Japan. ${ }^{5}$ Dept of Surgical Oncology, Research Institute for Radiation Biology and Medicine, Hiroshima University, Hiroshima, Japan. ${ }^{6}$ Dept of Thoracic Surgery, Koseikai Takeda Hospital, Kyoto, Japan. ${ }^{7}$ Hiroshima Cosmopolitan University, Hiroshima, Japan.

Correspondence: Noboru Hattori, Dept of Molecular and Internal Medicine, Graduate School of Biomedical and Health Sciences, Hiroshima University, 1-2-3 Kasumi, Minami-ku, Hiroshima 734-8551, Japan. E-mail: nhattoriahiroshima-u.ac.jp

@ERSpublications

Aminopeptidase $\mathrm{N}$ (APN)/CD13 is a potential therapeutic target in mesothelioma exhibiting high APN/CD13 expression http://ow.ly/dzmu30iGKzd

Cite this article as: Otsuki T, Nakashima T, Hamada H, et al. Aminopeptidase N/CD13 as a potential therapeutic target in malignant pleural mesothelioma. Eur Respir J 2018; 51: 1701610 [https://doi.org/ 10.1183/13993003.01610-2017].

ABSTRACT Angiogenesis is a crucial factor in the progression of malignant pleural mesothelioma (MPM) and antiangiogenic strategies might be effective against MPM. Aminopeptidase N (APN)/CD13 promotes tumour angiogenesis and is associated with poor prognosis; however, its clinical significance in MPM remains unclear.

In 37 consecutive patients with surgically resected MPM, we evaluated the association between immunohistochemical APN/CD13 expression in resected tumours and survival. Additionally, the antitumour and antiangiogenic effects of MT95-4, a fully humanised anti-APN/CD13 monoclonal antibody, were evaluated in mice orthotopically implanted with EHMES-10 (abundantly expressing APN/ CD13) and MSTO-211H (scarcely expressing APN/CD13) MPM cells.

High tumour APN/CD13 expression was associated with poor prognosis in MPM patients ( $\mathrm{p}=0.04)$, and MT95-4 treatment reduced tumour growth and angiogenesis in mice harbouring EHMES-10 but not MSTO-211H cells. Furthermore, in mice harbouring EHMES-10 cells, MT95-4 combined with cisplatin more effectively suppressed tumour progression than cisplatin alone.

Taken together, these results suggest that APN/CD13 is implicated in the aggressiveness of MPM. Here, MT95-4 treatment reduced tumour progression likely by inhibiting angiogenesis, suggesting APN/CD13 as a potential molecular target for MPM treatment. Additionally, combination treatment with MT95-4 and cisplatin could represent a promising approach to treating MPM exhibiting high APN/CD13 expression.

This article has supplementary material available from erj.ersjournals.com

Received: Aug 072017 | Accepted after revision: Feb 242018

Copyright OERS 2018 


\section{Introduction}

Malignant pleural mesothelioma (MPM) is a rare but aggressive malignant tumour with poor prognosis, with the number of MPM-related deaths continuing to increase worldwide $[1,2]$. Only a minority of patients with MPM are eligible for multimodality therapy, including surgery and radiotherapy, and effective therapeutic strategies for patients with inoperable MPM are limited; therefore, novel systemic therapies are needed.

Angiogenesis is a crucial factor in the progression of solid tumours and microvessel density is an independent prognostic factor of MPM [3-6], indicating that antiangiogenic strategies might be beneficial for MPM treatment. This is supported by a previous report showing that addition of bevacizumab to pemetrexed plus cisplatin treatment improved survival in patients with MPM [7].

Aminopeptidase $\mathrm{N}(\mathrm{APN}) / \mathrm{CD} 13$ is a $\mathrm{Zn}^{2+}$-dependent, membrane-bound ectopeptidase that catalyses the cleavage of N-terminal amino acids from small peptides. APN/CD13 consists of 967 amino acids with a short N-terminal cytoplasmic domain, a single transmembrane domain and a large extracellular domain, which contains the active site. APN/CD13 is widely expressed in mammalian cells, such as renal proximal tubular epithelial cells and myeloid progenitor cells, as well as in the small intestine epithelium and central nervous system synaptic membranes. Additionally, APN/CD13 is a multifunctional enzyme that is also involved in viral receptor function, cellular differentiation and antigen presentation [8-12]. Moreover, APN/CD13 is associated with tumour progression through the promotion of both tumour cell invasion and angiogenesis [13-18]. The expression of APN/CD13 in various types of tumours, including lung, pancreatic and colon cancers, as well as acute lymphoblastic leukaemia, has been reported [19-22]; however, the clinical significance of its expression in MPM remains to be elucidated. On the basis of these findings, we hypothesised that APN/CD13 is associated with MPM progression and is an attractive molecular target for the treatment of MPM. To determine the significance of APN/CD13 in MPM, we investigated correlations between APN/CD13 expression and patient survival by performing immunohistochemical staining of resected tumours in patients with surgically resected MPM. Additionally, to determine the efficacy of APN/CD13 as a molecular target in MPM treatment, we examined the antitumour and antiangiogenic effects of MT95-4, a fully humanised anti-APN/CD13 monoclonal antibody $(\mathrm{mAb})$, in an orthotopic implantation mouse model of MPM. Using this animal model, we also examined whether MT95-4 affected the antitumour effect of the cytotoxic agent cisplatin.

\section{Materials and methods}

Clinical and pathological characteristics of patients

We examined 37 consecutive patients with MPM who underwent pre-operative chemotherapy (cisplatin plus pemetrexed) and radical surgery at the Dept of Thoracic Surgery of Hiroshima University (Hiroshima, Japan) from February 2008 to August 2015. Major eligibility criteria for radical surgery were histologically confirmed diagnosis of MPM, including clinical subtype T0-3/N0-2/M0 disease, no prior treatment for the disease, age 20-75 years, Eastern Cooperative Oncology Group performance status 0 or 1 and predicted post-operative forced expiratory volume in $1 \mathrm{~s}>1000 \mathrm{~mL}$. By immunohistochemical staining, we investigated APN/CD13 expression in resected tumours obtained from patients with MPM. Six patients who died of MPM-unrelated causes were excluded. This study was approved by the Ethics Committees of Hiroshima University Hospital and conducted in accordance with the ethical standards established by the Helsinki Declaration of 1975. All patients gave informed consent in writing and permission to use their samples.

\section{APN/CD13 immunohistochemistry}

Formalin-fixed, paraffin-embedded tissue specimens were incubated with a mouse mAb against human APN/CD13 (Leica Biosystems, San Jose, CA, USA) at $4^{\circ} \mathrm{C}$ overnight, washed in PBS and incubated with a biotinylated rabbit anti-mouse IgG antibody (Vector Laboratories, Burlingame, CA, USA). The immunoreaction was amplified using a VECTASTAIN ABC kit (Vector Laboratories) and antibody binding was visualised with 3,3-diaminobenzidine solution acting as a chromogen. The sections were then counterstained with haematoxylin and dehydrated. Some sections were incubated with normal murine IgG as negative controls.

\section{Specimen classification based on immunohistochemical staining}

All immunostained sections were reviewed by two pathologists who were blind to the clinical status of the patients. The proportion of tumour cells showing high or low staining was determined in five random microscopic fields by counting individual tumour cells at high magnification. The APN/CD13 expression level was evaluated in at least 200 tumour cells at a magnification of $\times 400$. The mean proportion of APN/ $\mathrm{CD}_{13}{ }^{+}$tumour cells in the tumours was $17 \%$; therefore, we set $17 \%$ as a cut-off value to categorise tumours with high or low APN/CD13 expression. 


\section{Cells and culture}

Two human MPM cell lines, EHMES-10 and MSTO-211H, were used in this study. EHMES-10 cells were established from the pleural effusion of a patient with MPM at Ehime University (Matsuyama, Japan) $[23,24]$ and MSTO-211H cells were purchased from the American Type Culture Collection (Manassas, VA, USA). BALB/c mouse primary pulmonary artery endothelial cells were purchased from Cell Biologics (Chicago, IL, USA). MPM cell lines were cultured in RPMI 1640 medium (Gibco, Grand Island, NY, USA) supplemented with $10 \%$ fetal bovine serum and $1 \%$ penicillin/streptomycin. Mouse endothelial cells were cultured in Mouse Endothelial Cell Medium /w (Cell Biologics). All cells were cultured at $37^{\circ} \mathrm{C}$ in a $5 \%$ $\mathrm{CO}_{2}$ humidified atmosphere. MSTO-211H and mouse artery endothelial cells were used within 6 months after thawing, and EHMES-10 cells were authenticated by short tandem repeat analyses (Promega, Tokyo, Japan).

\section{Reagents and animals}

MT-95-4 was developed in our laboratory as previously reported [25] and cisplatin was purchased from Nippon Kayaku (Tokyo, Japan). Male severe combined immunodeficient (SCID) mice (6 weeks old) were purchased from CLEA Japan (Osaka, Japan). Animals were maintained according to guidelines for the ethical use of animals in research at Hiroshima University.

\section{Flow cytometric Analysis}

Flow cytometric analyses of MPM cell lines were performed as previously described [25].

\section{Quantitative real-time PCR}

RNA extraction, reverse transcription to CDNA and real-time quantitative PCR were performed as previously described [25]. The primers used were Hs00174265_m1 for APN/CD13, Hs00900055_m1 for vascular endothelial growth factor (VEGF) and 4352935E for $\beta$-actin (all from Applied Biosystems, Framingham, MA, USA).

\section{Orthotopic implantation model}

EHMES-10 $\left(3 \times 10^{6}\right)$ or MSTO- $211 \mathrm{H}\left(1 \times 10^{6}\right)$ cells were injected into the thoracic cavity of SCID mice as previously described [23] and the mice were randomly assigned to control or drug treatment groups. MT95-4 (0.3 or $1 \mathrm{mg} \cdot \mathrm{kg}^{-1}$ ) and control human IgG (Sigma-Aldrich, St Louis, MO, USA) were injected intraperitoneally twice weekly, and cisplatin $\left(3 \mathrm{mg} \cdot \mathrm{kg}^{-1}\right)$ was injected i.p. once weekly. All mice were killed on day 28 (EHMES-10) or day 21 (MSTO-211H) after tumour cell inoculation, the thoracic tumours were carefully removed and weighed, and pleural effusions were harvested using a $1 \mathrm{~mL}$ syringe, followed by volumetric measurement.

\section{Evaluation of microvessel density in thoracic tumours}

Microvessel density was evaluated following immunohistochemical staining for CD31 in thoracic tumours as previously described [26].

\section{Proliferation assay}

EHMES-10 or MSTO-211H $\left(1 \times 10^{4}\right.$ per $\left.100 \mathrm{~mL}\right)$ cells were seeded in 96-well plates and incubated with MT95-4 at various concentrations for $36 \mathrm{~h}$. Cell proliferation was assessed by measuring the absorbance of the medium in each well using the Cell Counting Kit-8 (Dojindo, Kumamoto, Japan).

In vivo analyses of apoptosis

Frozen tissue sections were used for in situ apoptosis detection by terminal deoxynucleotidyl transferase-mediated dUTP nick end-labelling (TUNEL) using the In Situ Apoptosis Detection kit (Takara Biomedicals, Shiga, Japan). Images were captured using a microscope at a magnification of $\times 200$ (BZ-9000; Keyence, Osaka, Japan) and the positive cells were determined by counting 1000 cells per slide.

Angiogenesis antibody array analysis

Angiogenesis antibody array analysis was performed as described in the supplementary material.

\section{Quantification of VEGF}

EHMES-10 $\left(1 \times 10^{5}\right)$ cells were seeded in 24-well plates and cultured in the presence of MT95-4 $\left(40 \mu \mathrm{g} \cdot \mathrm{mL}^{-1}\right)$ or human control IgG for 12 or $48 \mathrm{~h}$. The VEGF concentration in the culture supernatant was measured using an ELISA kit (R\&D Systems, Minneapolis, MN, USA) according to the manufacturer's instructions. 


\section{Matrix degradation assay}

A matrix degradation assay was performed using the Cell Invasion Assay kit (Cell Biolabs, San Diego, CA, USA) according to the manufacturer's instructions. In brief, cells were seeded on the upper surface of the insert membrane pre-coated with matrix proteins. Degradation of the matrix proteins was necessary for seeded cells to pass through the pores of the membrane. Finally, the invaded cells were stained and quantified. For mouse primary pulmonary artery endothelial cells, a suspension of $2 \times 10^{5}$ cells in various concentrations of recombinant human APN/CD13 was seeded on the upper surface of the insert membrane pre-coated with basement membrane matrix solution. For EHMES-10 and MSTO-211H cells, a suspension of $2 \times 10^{5}$ cells containing MT95-4 $\left(20 \mu \mathrm{g} \cdot \mathrm{mL}^{-1}\right)$ or human control IgG was seeded on the upper surface of the insert membrane pre-coated with matrix proteins. Invasive cells were counted in three random microscopic fields per well after $48 \mathrm{~h}$.

\section{Statistical analysis}

Fisher's exact test and the Mann-Whitney U-test were performed to determine significant differences between APN/CD13 expression and clinicopathological parameters. Overall survival was recorded from the date of surgery to the date of death due to MPM. The Kaplan-Meier method was used to estimate the probability of survival and significance was assessed by the log-rank test. Univariate and multivariate analyses of predictive factors for prognosis were performed using the Cox proportional hazard regression model to assess APN/CD13 expression as an independent prognostic marker for MPM. Independent predictors were identified from among the parameters whose $\mathrm{p}$-values were $<0.1$ in a univariate analysis. For in vitro experiments, data were expressed as the mean \pm SEM and differences between groups were analysed by the $t$-test or one-way ANOVA with Dunnett's post hoc test. For comparisons between groups in vivo, one-way ANOVA with Dunnett's post hoc test or Bonferroni's multiple comparison test was performed. Statistical analyses were performed using Prism version 6 (GraphPad, La Jolla, CA, USA) and JMP version 13.0 (SAS Institute, Cary, NC, USA). All p-values were recorded as two-tailed and $\mathrm{p}<0.05$ was considered statistically significant.

\section{Results}

\section{APN/CD13 expression is associated with shorter overall survival in MPM patients}

To investigate the clinical significance of APN/CD13 in MPM, we immunohistochemically evaluated APN/ CD13 expression in resected tumours and analysed its association with survival in MPM patients. Representative images of positive and negative immunohistochemical staining of APN/CD13 in resected tumours are shown in figure 1a and b, respectively. We observed APN/CD13 staining throughout the cell membrane and cytoplasm of tumour cells, and 15 and 16 tumours were categorised as exhibiting high and low APN/CD13 expression, respectively. The relationships between APN/CD13 expression and clinicopathological variables are shown in table 1 . The median (range) age at surgery was 64 (48-72) years and 19 patients died during the follow-up period. No significant relationships between APN/CD13 expression and age, smoking history, effect of neoadjuvant chemotherapy or histology were observed; however, we could not investigate the relationship between APN/CD13 expression and sex because female patients were not included. The median survival time of patients with high levels of APN/CD13 expression was significantly shorter than that of patients with low levels of APN/CD13 expression (17.3 versus 54.4 months; $\mathrm{p}=0.04$ ) (figure 1c). Furthermore, to investigate APN/CD13 as an independent prognostic marker, univariate and multivariate analyses were performed using the Cox proportional hazard regression model. A multivariate analysis demonstrated that both high APN/CD13 expression and histology (nonepithelioid) were independently associated with poor prognosis among the factors with $\mathrm{p}<0.1$ in a univariate analysis (table 2).

\section{MT95-4 suppresses tumour progression in an APN/CD13 expression-dependent manner}

To determine the efficacy of APN/CD13 as a molecular target in MPM, we examined the antitumour effects of MT95-4, a fully humanised anti-APN/CD13 mAb, in an orthotopic implantation mouse model of MPM, using the MPM cell lines EHMES-10 and MSTO-211H. Both quantitative real-time PCR and flow cytometric analysis showed that EHMES-10 cells abundantly expressed APN/CD13, whereas MSTO-211H cells scarcely expressed APN/CD13 (figure 2a and b). In vivo administration of MT95-4 at doses of 0.3 and $1.0 \mathrm{mg} \cdot \mathrm{kg}^{-1}$ significantly reduced tumour weight (ANOVA; $\mathrm{p}=0.03$ and $\mathrm{p}=0.01$, respectively). Additionally, administration of MT95-4 at $1.0 \mathrm{mg} \cdot \mathrm{kg}^{-1}$ significantly reduced the amount of pleural effusion (ANOVA; $p=0.02$ ) in mice harbouring EHMES-10 cells (figure $2 c$ and $d$ ); however, in mice harbouring MSTO-211H cells, MT95-4 administration reduced neither tumour weight nor the amount of pleural effusion (figure $2 \mathrm{e}$ and $\mathrm{f}$ ). 

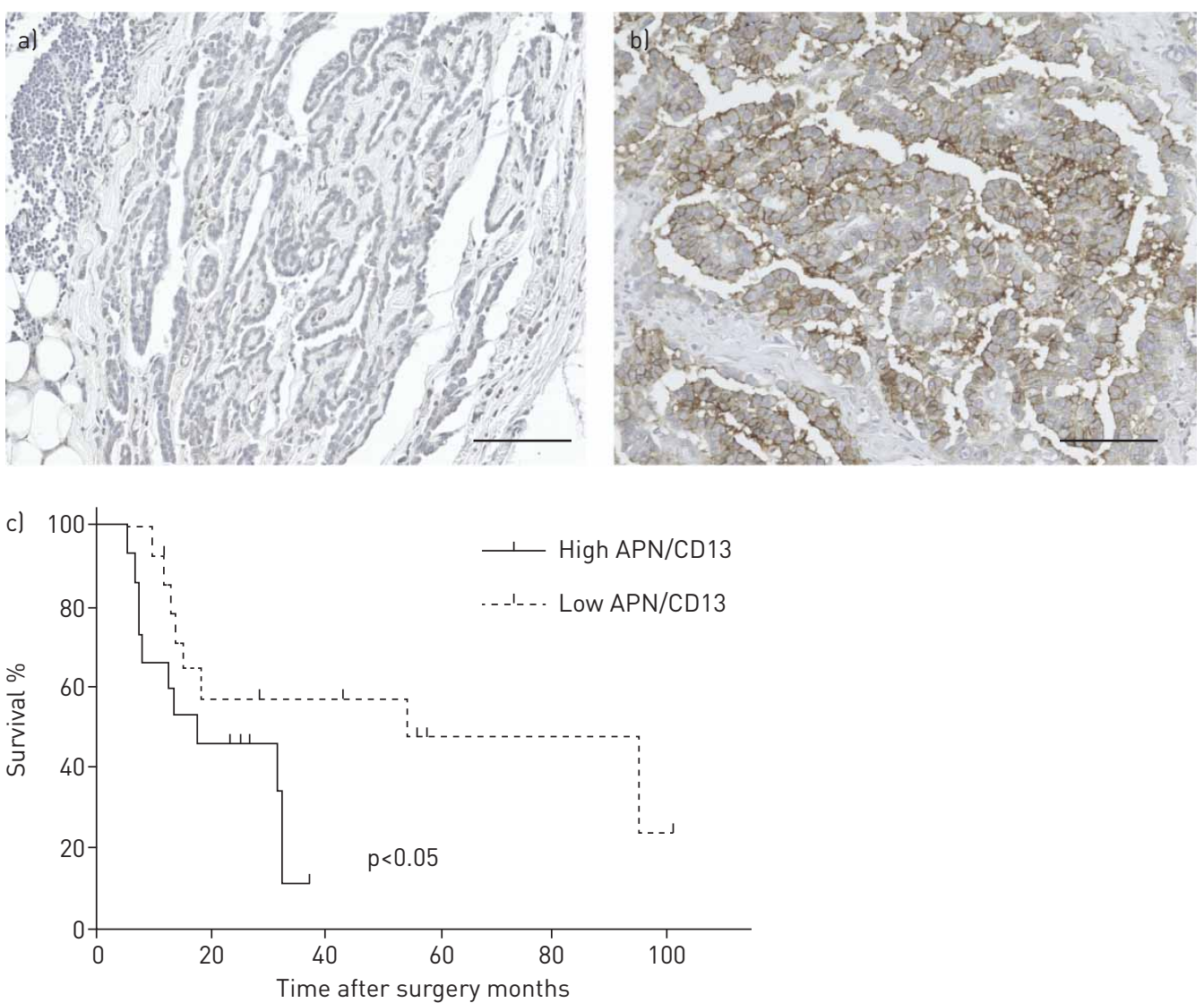

FIGURE 1 Immunohistochemical staining for aminopeptidase N (APN)/CD13 in tumour tissues operatively obtained from patients with malignant pleural mesothelioma (MPM). a) Representative image of negative APN/CD13 staining. b) Representative image of positive APN/CD13 staining. Scale bar: $100 \mu \mathrm{m}$. c) Overall survival of patients with MPM in relation to the status of APN/CD13 expression in tumours. Censored patients are indicated by vertical marks. p-value determined by the log-rank test.

TABLE 1 Relationships between aminopeptidase N (APN)/CD13 expression and various clinical and pathological variables

\begin{tabular}{|c|c|c|c|c|}
\hline & \multirow[t]{2}{*}{ Total } & \multicolumn{2}{|c|}{ APN/CD13 } & \multirow[t]{2}{*}{$p$-value } \\
\hline & & High & Low & \\
\hline \multicolumn{5}{|l|}{ Sex } \\
\hline Female & 0 & 0 & 0 & $>0.999^{\#}$ \\
\hline Male & 31 & 15 & 16 & \\
\hline Age years & $64(48-72)$ & $64(55-72)$ & $63(48-70)$ & \\
\hline \multicolumn{5}{|l|}{ Smoking status } \\
\hline Never-smoker & 5 & 3 & 2 & $0.654^{\#}$ \\
\hline Smoker & 26 & 12 & 14 & \\
\hline \multicolumn{5}{|c|}{ Effect of neoadjuvant chemotherapy } \\
\hline Partial remission & 14 & 7 & 7 & $>0.999^{\natural}$ \\
\hline Stable disease & 17 & 8 & 9 & \\
\hline \multicolumn{5}{|l|}{ Histology } \\
\hline Epithelioid & 23 & 10 & 13 & $0.591^{\pi}$ \\
\hline Biphasic & 7 & 5 & 2 & \\
\hline Sarcomatoid & 1 & 0 & 1 & \\
\hline Total & 31 & 15 & 16 & \\
\hline
\end{tabular}

Data are presented as $\mathrm{n}$ or median (range), unless otherwise stated. ": Fisher's exact test; ": MannWhitney U-test. 


\begin{tabular}{|c|c|c|}
\hline & Hazard ratio $(95 \% \mathrm{CI})$ & p-value \\
\hline \multicolumn{3}{|l|}{ Univariate analysis } \\
\hline Age $>64$ years & $2.415(0.75281-8.34558)$ & 0.137 \\
\hline High APN/CD13 expression & $3.943(1.05241-19.11679)$ & 0.0416 \\
\hline Effect of neoadjuvant chemotherapy (stable disease) & $1.496(0.51525-4.58769)$ & 0.4572 \\
\hline Smoker & $0.361(0.07528-2.56382)$ & 0.2679 \\
\hline Histology (nonepithelioid) & $6.299(1.03425-48.1547)$ & 0.0461 \\
\hline \multicolumn{3}{|l|}{ Multivariate analysis } \\
\hline High APN/CD13 expression & 4.088 (1.08272-19.88195) & 0.0375 \\
\hline Histology (nonepithelioid) & $6.748(1.08035-52.63304)$ & 0.0416 \\
\hline
\end{tabular}

MT95-4 suppresses tumour angiogenesis in an APN/CD13 expression-dependent manner To investigate the antiangiogenic effect of MT95-4 in intrapleural tumours, we evaluated the microvessel density of intrapleural tumours in mice harbouring EHMES-10 or MSTO-211H cells by immunohistochemical staining for CD31. MT95-4 administration significantly decreased microvessel density (ANOVA; all p<0.001) in intrapleural tumours in mice harbouring EHMES-10 cells (figure 3a and b), but not in mice harbouring MSTO- $211 \mathrm{H}$ cells (figure $3 \mathrm{c}$ and $\mathrm{d}$ ).

\section{MT95-4 enhances the antitumour effect of cisplatin in mouse models of MPM}

As the antitumour effect of MT95-4 was demonstrated in mice harbouring MPM cells exhibiting high levels of APN/CD13 expression, we investigated whether MT95-4 administration affected the antitumour effect of the cytotoxic agent cisplatin. Mice harbouring EHMES-10 cells were treated with vehicle, MT95-4 alone, cisplatin alone or MT95-4 and cisplatin combination therapy. As shown in figure 4a and b, MT95-4 and cisplatin combination therapy significantly reduced both tumour weight (ANOVA; $\mathrm{p}=0.009$ ) and the amount of pleural effusion (ANOVA; $\mathrm{p}=0.04$ ) compared with cisplatin alone. Moreover, MT95-4 and cisplatin combination therapy significantly prolonged the survival time of mice harbouring EHMES-10 cells (figure 4c).

Effects of MT95-4 on the degree of proliferation and apoptosis

MT95-4 had an antitumour effect by inhibiting angiogenesis in vivo; we further investigated other mechanisms underlying the antitumour effect. A proliferation assay showed that MT95-4 did not suppress the proliferation rate of both EHMES-10 and MSTO- $211 \mathrm{H}$ cells in vitro (figure $5 \mathrm{a}$ and $\mathrm{b}$ ). Next, we evaluated apoptosis in situ by TUNEL using frozen tissue sections and found that MT95-4 alone did not significantly increase the number of apoptotic cells. However, MT95-4 in combination with cisplatin did significantly increase the number of apoptotic cells (figure $5 \mathrm{c}$ and $\mathrm{d}$ ).

\section{Antiangiogenic effect of MT95-4 depends on the inhibition of extracellular matrix degradation, but not the inhibition of angiogenic factors}

As the antiangiogenic activity of MT95-4 was demonstrated in vivo, we explored the mechanism underlying the antiangiogenic effects of MT95-4. We first investigated whether MT95-4 directly affected the expression of angiogenic factors produced by tumour cells in vitro. Using an angiogenesis antibody array, the expression levels of 43 angiogenic factors were compared between EHMES-10 cells treated with or without MT95-4. As shown in figure 6a, treatment of EHMES-10 cells with MT95-4 did not affect the expression of angiogenic factors. To verify these findings, we evaluated the protein and mRNA levels of the most important angiogenic factor, i.e. VEGF, in EHMES-10 cells treated with MT95-4. Our results showed that treatment of EHMES-10 cells with MT95-4 did not affect either VEGF protein or mRNA levels (figure $6 \mathrm{~b}$ and $\mathrm{c}$ ). Given that the angiogenic factor did not play a key role in the antiangiogenic effect of MT95-4, we hypothesised that the antiangiogenic effect of MT95-4 in vivo was achieved by the inhibition of extracellular matrix degradation, thereby inhibiting the invasion of vascular endothelial cells into the perivascular stroma. To evaluate this hypothesis, we performed a matrix degradation assay. First, to assess whether MT95-4 suppresses extracellular matrix degradation via the blockade of APN/CD13 of mesothelial cells, a matrix degradation assay was performed using a EHMES-10 (abundantly expressing APN/CD13) or MSTO-211H (scarcely expressing APN/CD13) cell suspension containing MT95-4 $\left(20 \mu \mathrm{g} \cdot \mathrm{mL}^{-1}\right)$ or human control IgG. MT95-4 suppressed the invasion of EHMES-10, but not MSTO-211H (figure $6 \mathrm{~d}$ and e). Second, we investigated whether APN/CD13 directly promotes the 

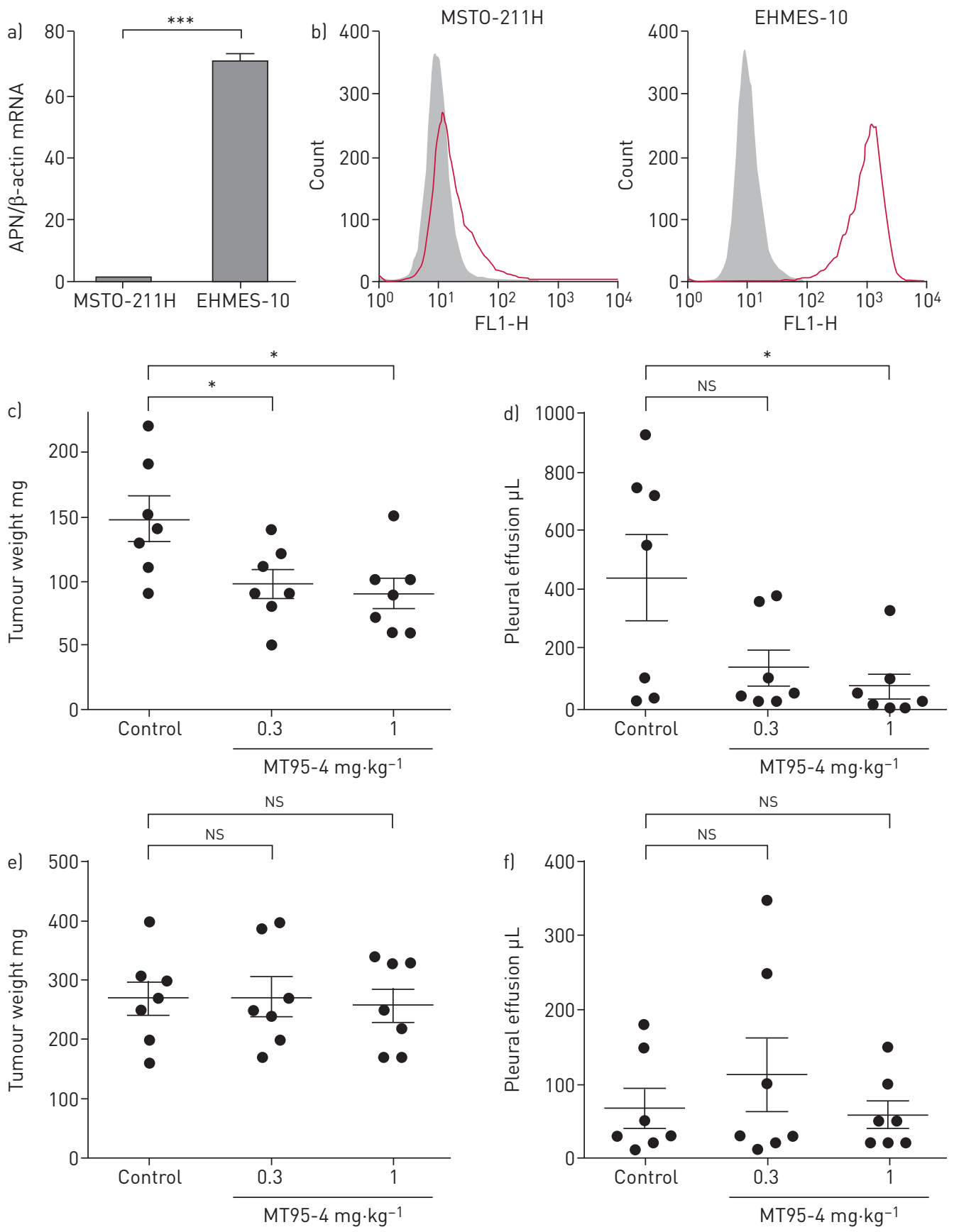

FIGURE 2 In vivo effect of MT95-4 on thoracic tumour weight and pleural effusion in an orthotopic implantation model of malignant pleural mesothelioma (MPM). a) Expression levels of aminopeptidase $\mathrm{N}$ (APN)/CD13 mRNA in human MPM cells analysed by quantitative real-time PCR. Data are presented as mean \pm SEM of triplicate samples. ${ }^{* * *}: p<0.001$. b) APN/CD13 expression on the surface of human MPM cells analysed by flow cytometry. c-f) Evaluation of tumour weight and pleural effusion produced by MPM cells in severe combined immunodeficient (SCID) mice using c, d) EHMES-10 or e, f) MSTO-211H cells. EHMES-10 $\left(3 \times 10^{6}\right)$ or MSTO- $211 \mathrm{H}\left(1 \times 10^{6}\right)$ cells were inoculated into the thoracic cavity of SCID mice, followed by treatment with MT95-4 (0.3 or $\left.1 \mathrm{mg} \cdot \mathrm{kg}^{-1}\right)$ or an isotype-matched control monoclonal antibody i.p. injected twice weekly. Thoracic tumour weight and pleural effusion were evaluated at 4 weeks (EHMES-10) or 3 weeks (MSTO-211H) after tumour cell inoculation. Horizontal lines indicate the mean \pm SEM associated with seven mice per group. Data were analysed by one-way ANOVA with Dunnett's post hoc test. *: $p<0.05$ versus control group; NS: not significant.

invasion of mouse vascular endothelial cells by the degradation of the matrix proteins. A matrix degradation assay using mouse primary pulmonary artery endothelial cell suspensions containing various concentrations of recombinant human APN/CD13 showed that recombinant human APN/CD13 promoted the invasion of endothelial cells in a dose-dependent manner (figure $6 \mathrm{f}$ ). 


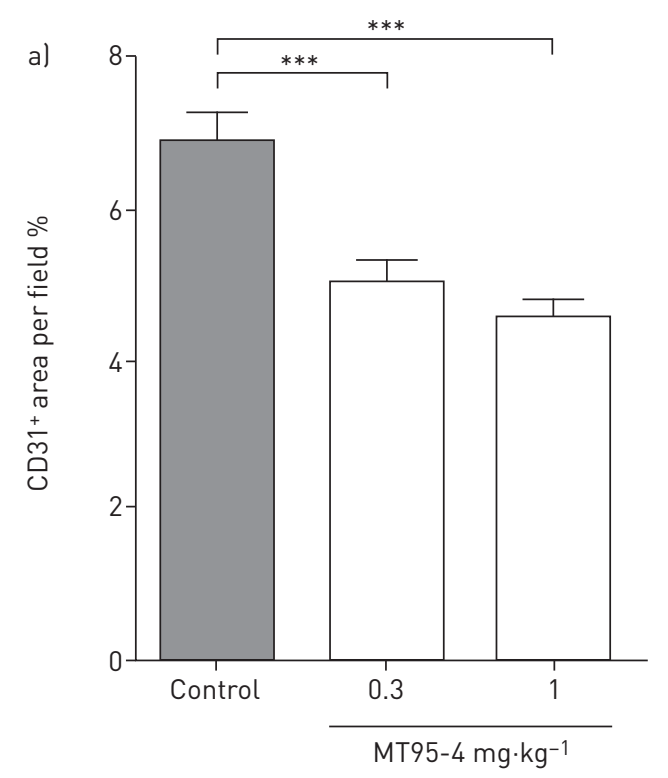

b)
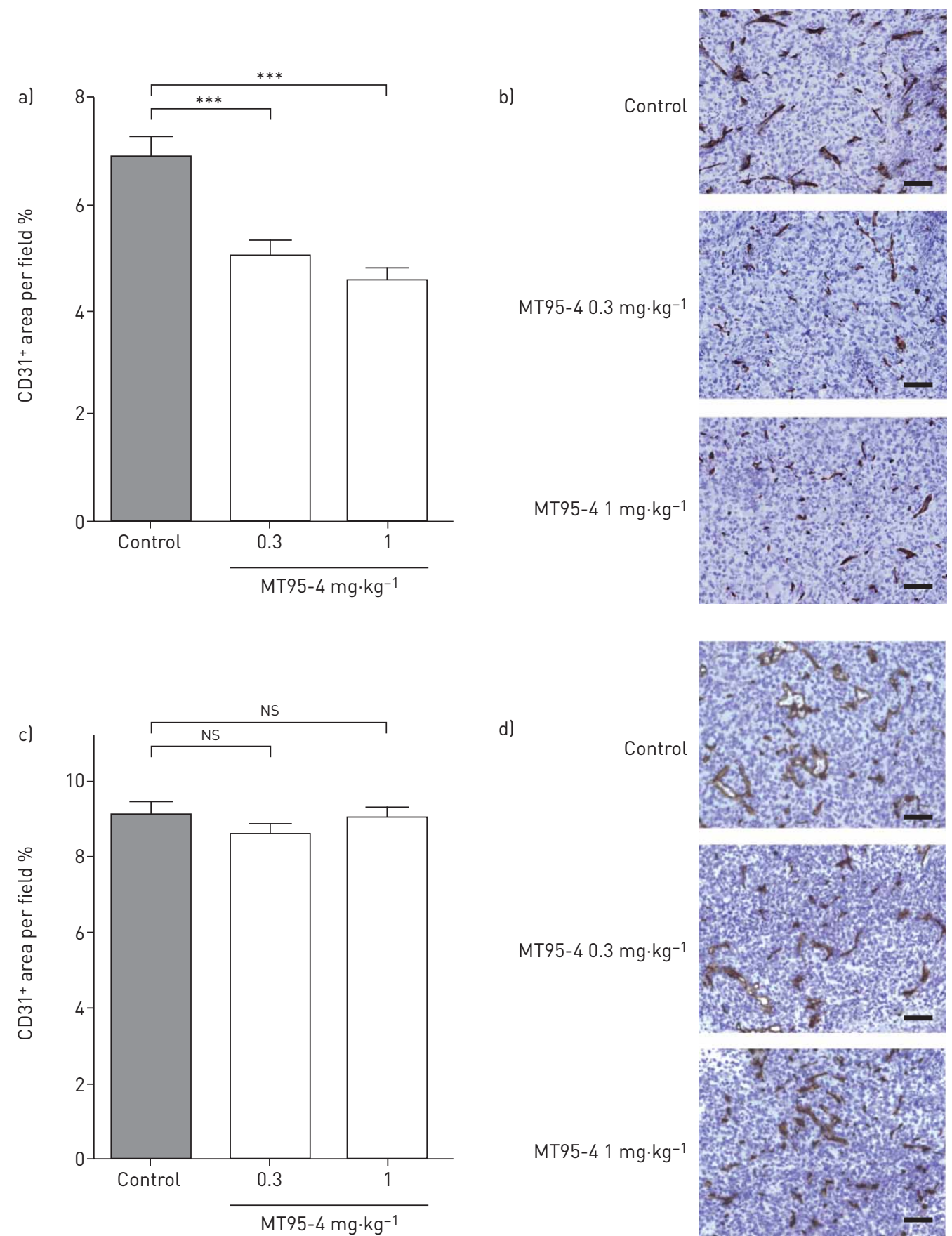

d)

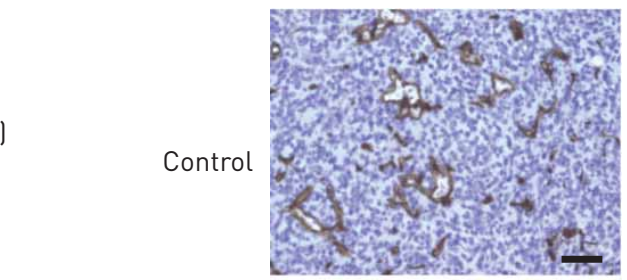

MT95-4 $0.3 \mathrm{mg} \cdot \mathrm{kg}^{-1}$
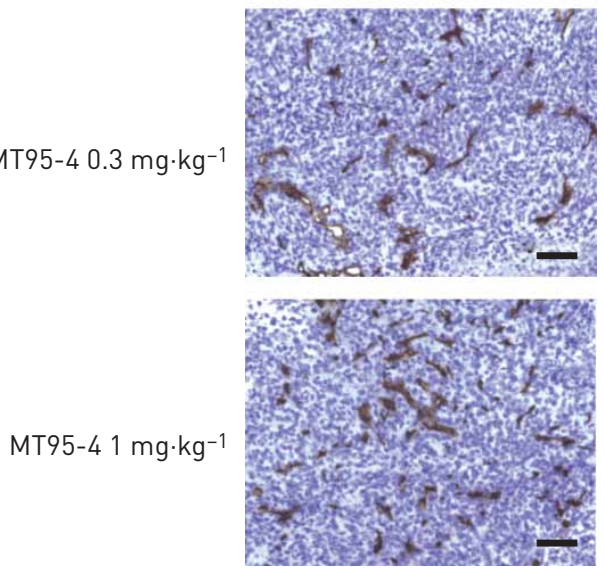

FIGURE 3 Effect of MT95-4 treatment on angiogenesis in thoracic tumours. a-d) Mice transplanted with a, b) EHMES-10 or c, d) MSTO-211 H cells were treated with MT95-4 $\left(0.3\right.$ or $\left.1 \mathrm{mg} \cdot \mathrm{kg}^{-1}\right)$ or an isotype-matched control monoclonal antibody by i.p. injection twice weekly. The area of CD31 ${ }^{+}$vessels in mice transplanted with a) EHMES-10 or c) MSTO-211 H cells was calculated as described in the Materials and methods. $n=7$ per group. Data were analysed by one-way ANOVA with Dunnett's post hoc test. $* * *$ : $p<0.001$ versus control group; NS: not significant. $b, d$ I Immunohistochemical staining for CD31 in thoracic tumours derived from b) EHMES-10 or d) MSTO-211H cells. Scale bar: $100 \mu \mathrm{m}$.

\section{Discussion}

In this study, we found that high APN/CD13 expression was potentially associated with poor prognosis in MPM patients, and demonstrated that MT95-4, a fully humanised anti-APN/CD13 mAb, exerted antitumour and antiangiogenic effects in mice harbouring EHMES-10 cells (abundantly expressing APN/ CD13), but not in those harbouring MSTO-211H cells (scarcely expressing APN/CD13). In mice harbouring EHMES-10 cells, administration of MT95-4 in combination with cisplatin suppressed tumour progression more effectively and prolonged survival compared with treatment with cisplatin alone. 

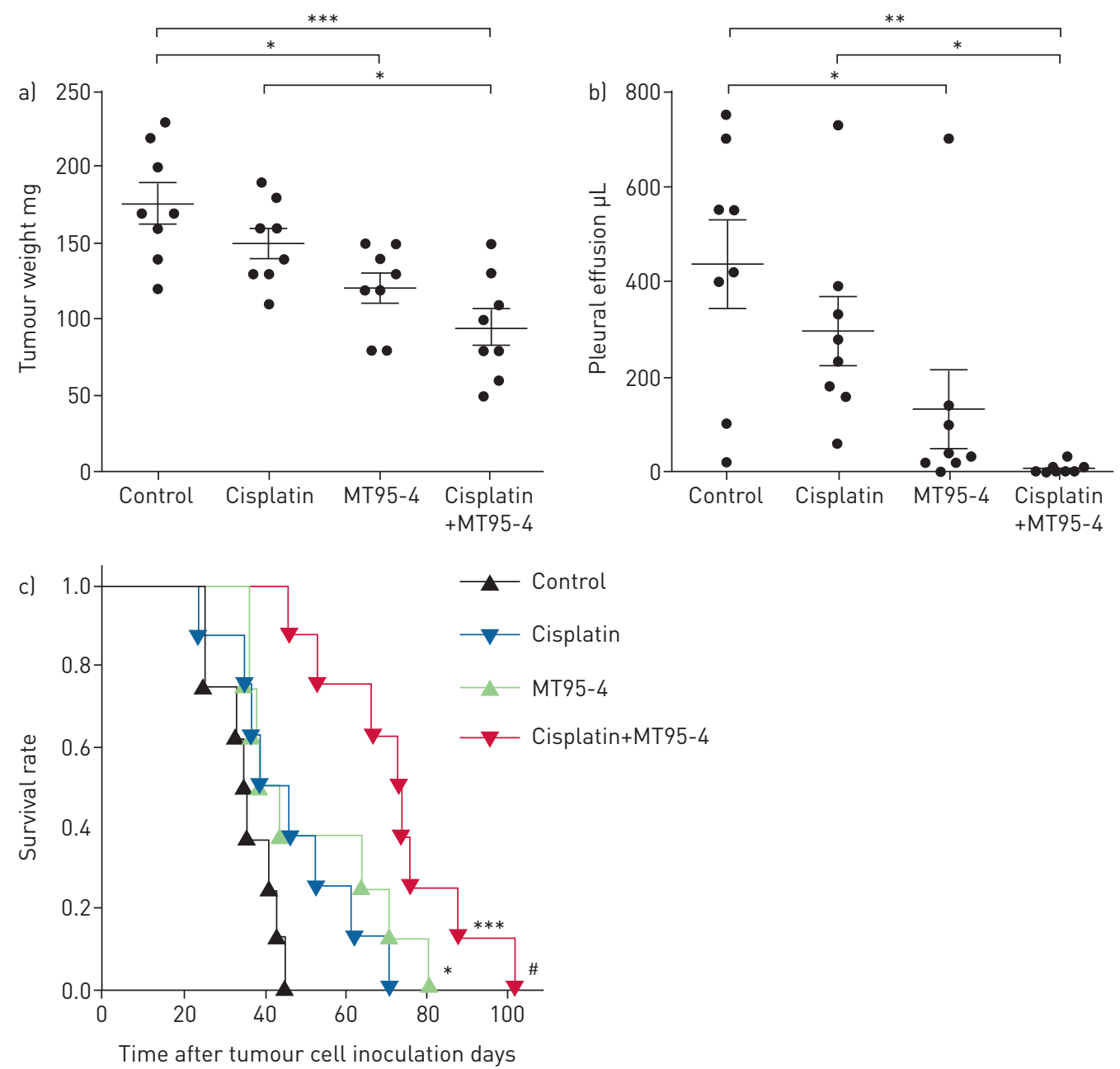

FIGURE 4 In vivo effect of MT95-4 treatment in combination with cisplatin in an orthotopic implantation model of malignant pleural mesothelioma using EHMES-10 cells. a, b) Effect of MT95-4 treatment in combination with cisplatin on a) tumour weight and b) pleural effusion in severe combined immunodeficient (SCID) mice harbouring EHMES-10 cells. EHMES-10 $\left(3 \times 10^{6}\right)$ cells were inoculated into the thoracic cavity of SCID mice, followed by treatment with MT95-4 $\left(0.3 \mathrm{mg} \cdot \mathrm{kg}^{-1}\right.$ i.p. twice weekly), cisplatin $\left(3 \mathrm{mg} \cdot \mathrm{kg}^{-1}\right.$ i.p. once weekly) or MT95-4 $\left(0.3 \mathrm{mg} \cdot \mathrm{kg}^{-1}\right)$ in combination with cisplatin $\left(3 \mathrm{mg} \cdot \mathrm{kg}^{-1}\right)$. Thoracic tumour weight and pleural effusion were evaluated 4 weeks after tumour cell inoculation. Horizontal lines indicate the mean \pm SEM associated with eight mice per group. Data were analysed by one-way ANOVA and Bonferroni's multiple comparison test. *: $p<00.05 ;{ }^{* *}$ : $p<0.01 ;{ }^{* *}: p<0.001$. c) Effect of MT95-4 treatment in combination with cisplatin on survival of mice harbouring EHMES-10 cells. $p$-values were determined by the log-rank test. ${ }^{*}: p<0.05$ versus control group; $^{* * *}: p<0.001$ versus control group; ${ }^{*}: p<0.01$ versus cisplatin monotherapy group.

Additionally, in vitro experiments demonstrated that the antiangiogenic effect of MT95-4 might be mediated by the inhibition of extracellular matrix degradation and thereby the inhibition of vascular endothelial cell invasion, but not by the suppression of angiogenic factors produced by tumour cells.

Our results clearly show that high APN/CD13 expression in tumour cells was associated with poor prognosis in MPM patients. Although APN/CD13 expression is associated with poor prognosis in various tumour types [19-22], possibly through its ability to promote tumour cell invasion and angiogenesis [13-18], its clinical significance in MPM remains unknown. To the best of our knowledge, this represents the first study reporting an association between APN/CD13 expression in tumour cells and MPM patient prognosis. These results indicate that APN/CD13 is involved in tumour aggressiveness in MPM patients.

To examine the efficacy of APN/CD13 as a therapeutic target in MPM, we tested the antitumour effect of MT95-4, a fully humanised anti-APN/CD13 mAb established in our laboratory [25], in an orthotopic implantation mouse model of MPM, using MPM cells exhibiting high or low APN/CD13 expression. We found that MT95-4 administration suppressed tumour progression and angiogenesis only in tumours exhibiting high APN/CD13 expression. These results suggest that a therapeutic strategy targeting APN/ CD13 might constitute a promising treatment option for MPM exhibiting high APN/CD13 expression. 


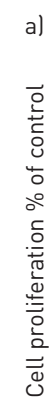

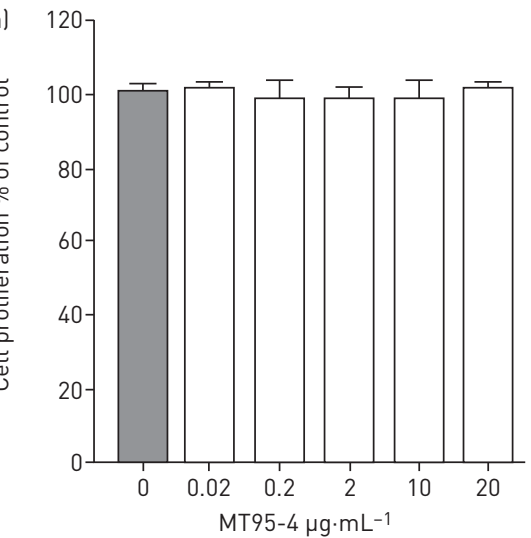

c)
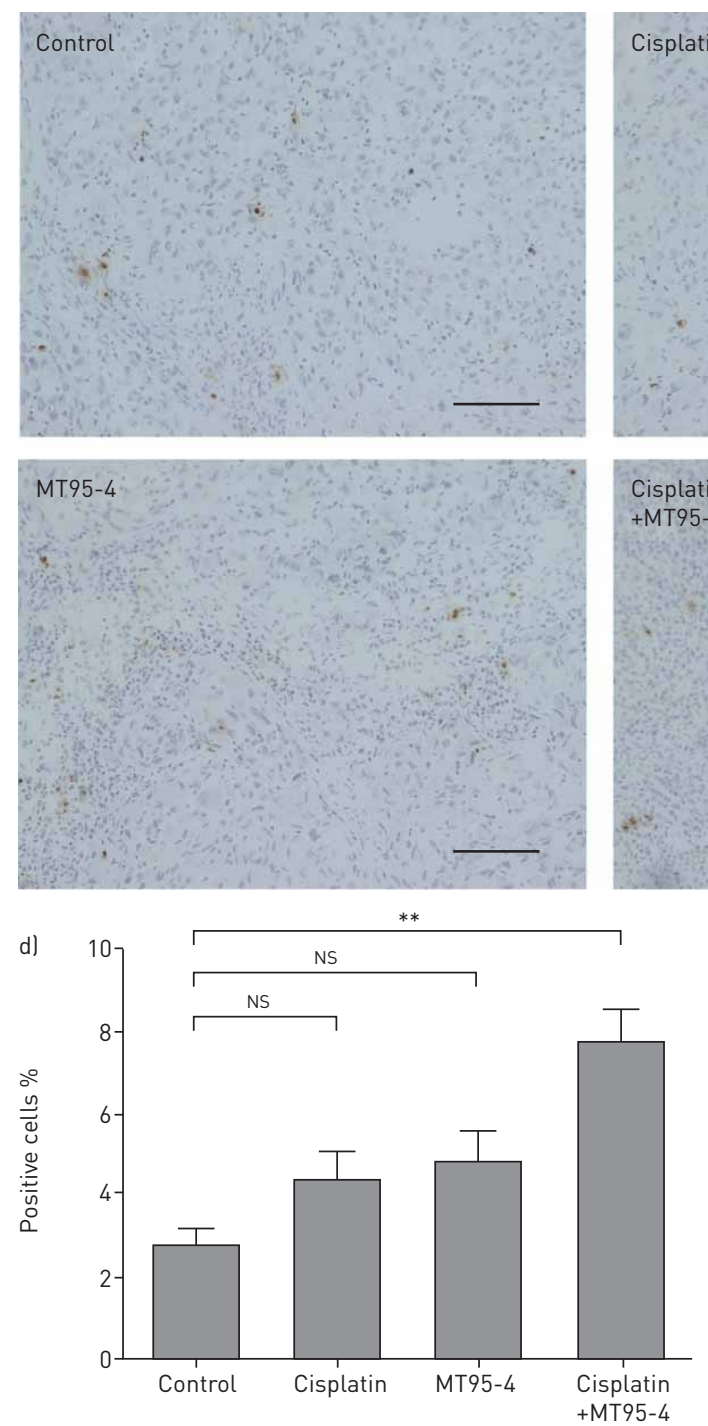

MT95-4
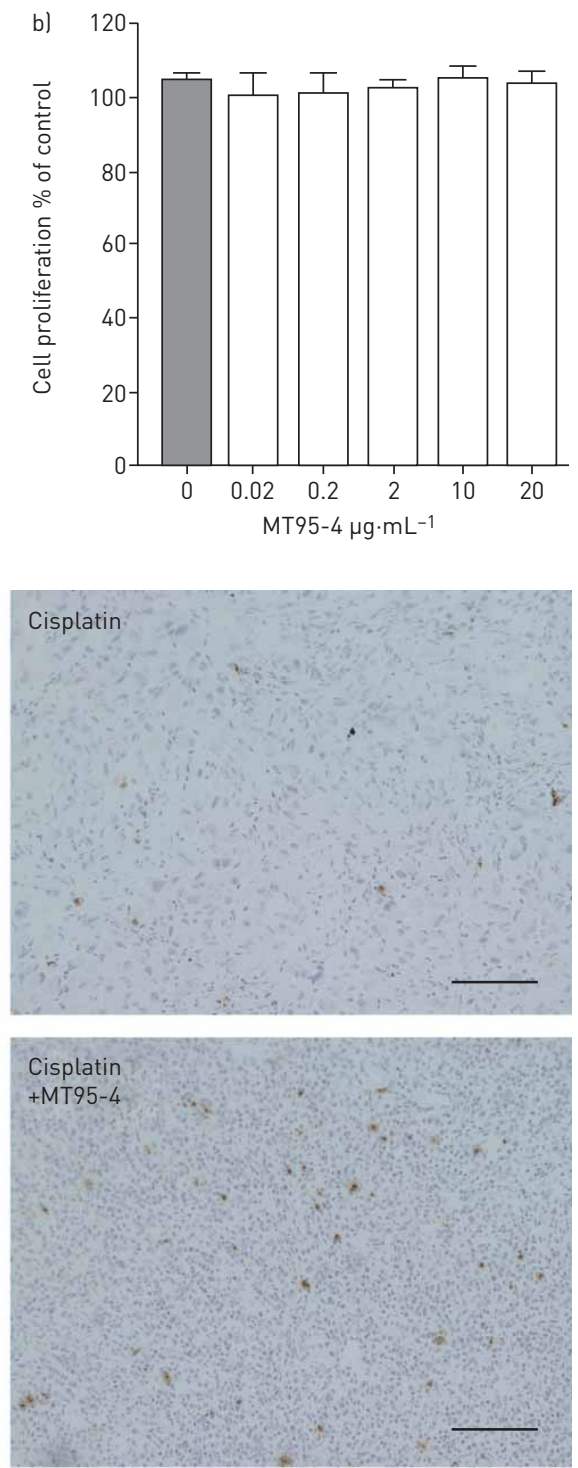

FIGURE 5 Effects of MT95-4 on the degree of proliferation and apoptosis. a, b) Proliferation was quantified in a) EHMES-10 and b) MSTO-211 H cells after $36 \mathrm{~h}$ in the presence of various concentrations of MT95-4. Data are presented as the mean \pm SEM of triplicate samples. C, d) In vivo analyses of apoptosis in thoracic tumours produced by EHMES-10 cells. Mice harbouring EHMES-10 cells were treated with MT95-4 $10.3 \mathrm{mg} \cdot \mathrm{kg}^{-1}$ i.p. twice weekly), cisplatin ( $3 \mathrm{mg} \cdot \mathrm{kg}^{-1}$ i.p. once weekly) or MT95-4 $\left(0.3 \mathrm{mg} \cdot \mathrm{kg}^{-1}\right)$ in combination with cisplatin $\left(3 \mathrm{mg} \cdot \mathrm{kg}^{-1}\right)$. Four weeks after tumour cell inoculation, the mice were sacrificed. c) Thoracic tumours were evaluated for apoptosis by terminal deoxynucleotidyl transferase-mediated dUTP nick end-labelling. The positive cells were calculated as described in the Materials and methods. $n=3$ per group. Scale bar: $100 \mu \mathrm{m}$. d) Quantitative immunohistochemical analysis. Data are presented as the mean \pm SEM and were analysed by one-way ANOVA with Dunnett's post hoc test. ${ }^{* *}: p<0.01$ versus control group; Ns: not significant. 
ELISA $12 \mathrm{~h}$

a)
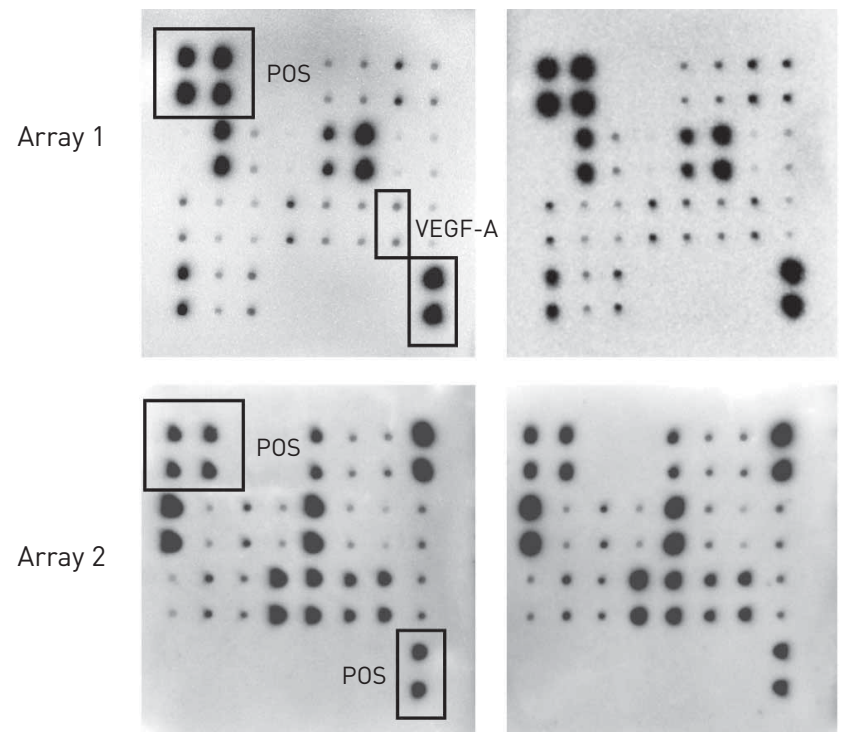

MT95-4
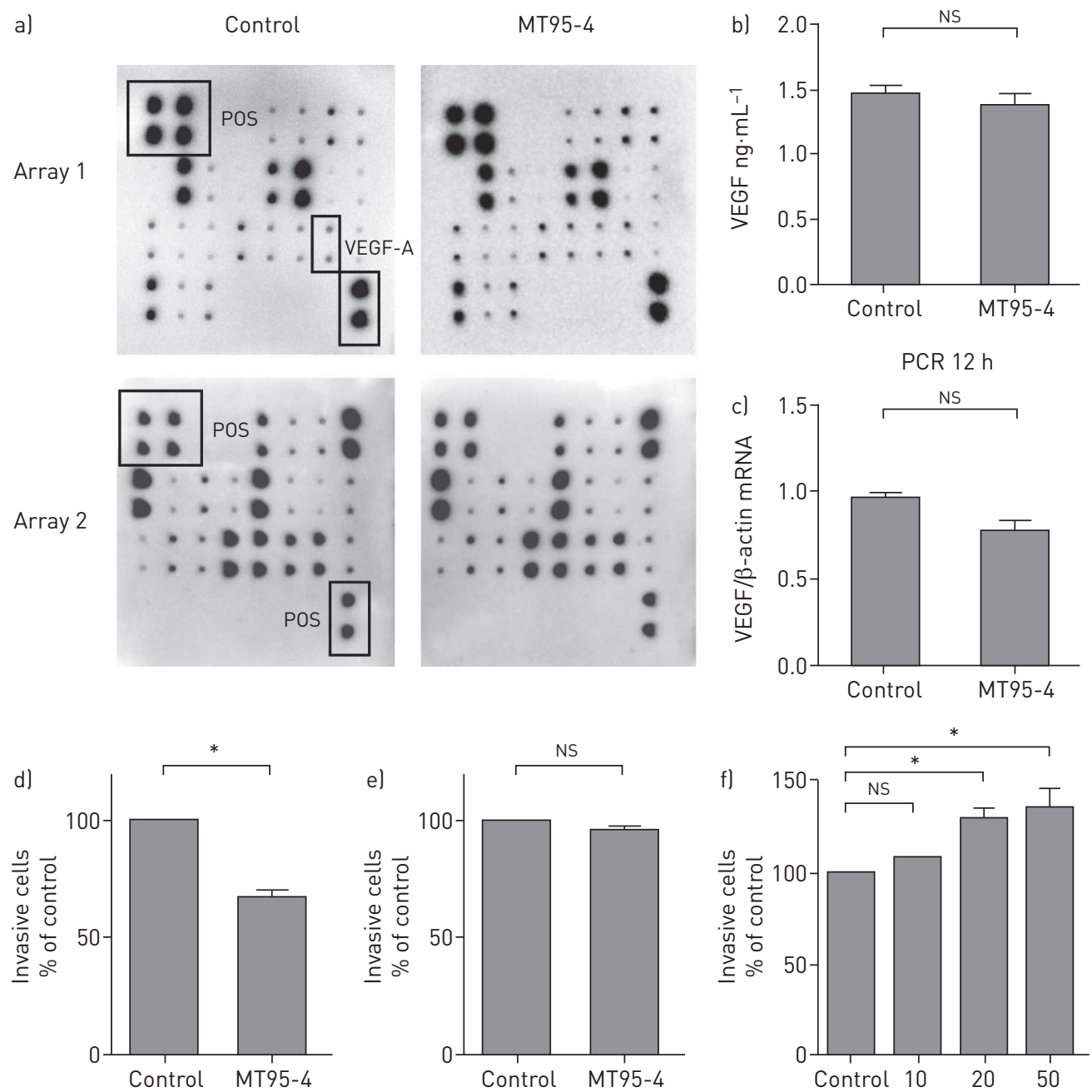

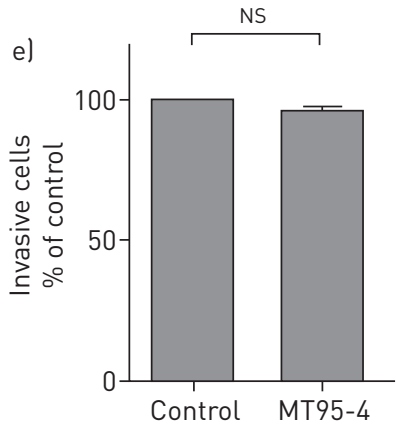

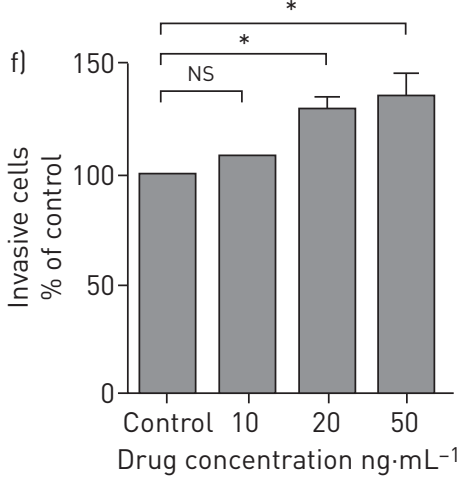

ELISA $48 \mathrm{~h}$
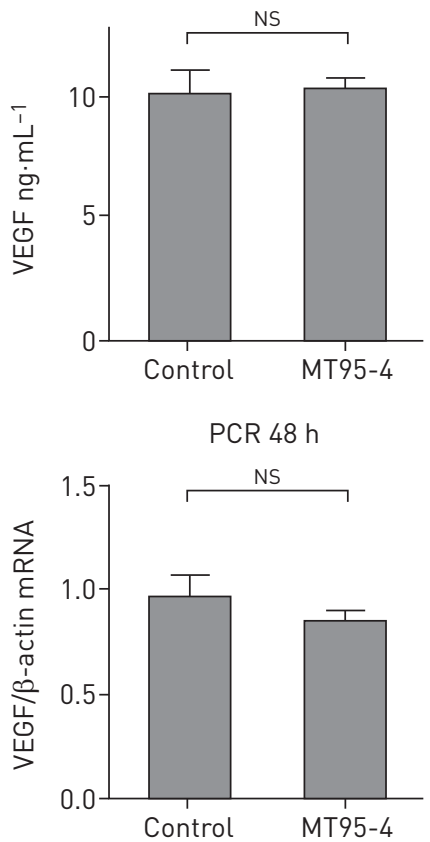

FIGURE 6 Antiangiogenic effect of MT95-4 depended on the inhibition of matrix degradation, but not the inhibition of angiogenic factors. a) Angiogenesis antibody array analysis of EHMES-10 cells treated with MT95-4 or human control IgG. POS: positive control; VEGF: vascular endothelial growth factor. b, c) EHMES-10 cells $\left(1 \times 10^{5}\right)$ were seeded on 24-well plates and cultured with MT95-4 $\left(40 \mu \mathrm{g} \cdot \mathrm{mL}^{-1}\right)$ or human control IgG for $24 \mathrm{~h}$. The densities of individual spots were visualised using a chemiluminescence detection system. b) VEGF concentrations in culture media were measured by ELISA and c) VEGF mRNA levels were analysed by quantitative real-time PCR. d, e) Effects of MT95-4 on the invasion of d) EHMES-10 and e) MSTO-211 H cells in the matrix degradation assay. f) Effect of recombinant human aminopeptidase N/CD13 on the invasion of mouse primary pulmonary artery endothelial cells. The number of invasive cells was counted in three random microscopic fields per well. Data are presented as mean \pm SEM of triplicate samples and were analysed by the t-test or one-way ANOVA with Dunnett's post hoc test. *: p<0.05 versus control group; NS: not significant.

As MT95-4 treatment alone did not affect cell proliferation (figure 5a and b), apoptosis (figure $5 \mathrm{c}$ and d) or senescence (data not shown), we suggest that MT95-4 suppresses tumour progression mainly by inhibiting angiogenesis in MPM tumours exhibiting high APN/CD13 expression.

A previous study reported that addition of bevacizumab to pemetrexed plus cisplatin treatment significantly improved overall survival in patients with unresectable MPM [7], thereby reaffirming the importance of antiangiogenic therapy in MPM. In this study, we demonstrated an antiangiogenic effect of neutralisation of APN/CD13 expression in tumour cells in an MPM mouse model harbouring MPM cells exhibiting high APN/CD13 expression. This result agreed with those of previous studies reporting that APN/CD13 neutralisation or knockdown in tumour cells decreased intratumoural microvessel density in mice $[25,27]$. Bevacizumab administration inhibits the activity of VEGF secreted by tumour cells; however, our results demonstrated that MT95-4 treatment did not suppress VEGF expression in MPM cells. Additionally, use of an angiogenic antibody array enabled us to observe that MT95-4 treatment did not affect the expression levels of 43 angiogenic factors, including VEGF, produced by MPM cells. These results suggest that the antiangiogenic effect associated with MT95-4 treatment was not mediated by the 
suppression of angiogenic factors. A matrix degradation assay showed that the invasiveness of vascular endothelial cells was enhanced in the presence of APN/CD13 and MT95-4 suppressed extracellular matrix degradation by tumour cells by inhibiting APN/CD13 activity. Consequently, we suggest that the antiangiogenic effect of MT95-4 treatment was achieved by the inhibition of APN/CD13 activity, which is involved in the degradation of extracellular matrix elements, such as entactin and type IV collagen (components of the basement membrane) $[13,28]$, thereby inhibiting the release and migration of vascular endothelial cells into the perivascular stroma [29, 30].

APN/CD13 expressed in both tumour cells and nonmalignant stromal cells promotes tumour progression and angiogenesis [27]. As MT95-4 does not recognise murine APN/CD13 [25], the antitumour and antiangiogenic effects of MT95-4 described in this study might have been achieved by inhibiting the activity of APN/CD13 expressed only in tumour cells. When used for patients with MPM, MT95-4 may exert stronger antitumour effects through inhibition of APN/CD13 activity in both tumour and stromal cells. Although APN/CD13 expression is not limited to the tumour microenvironment, a therapeutic approach involving MT95-4 targeting APN/CD13 should be feasible in clinical use. Notably, APN/CD13 deficiency in mice does not result in physiological or histological abnormalities [31] and ubenimex, an APN/CD13 inhibitor, has been used in clinical practice without severe adverse effects [32]. Additionally, we found that MT95-4 treatment in combination with cisplatin, a fundamental cytotoxic agent used for MPM treatment, exerted stronger antitumour effects compared with MT95-4 treatment alone in a mouse model of MPM. It was previously reported that CD13-overexpressing xenografts exhibited a reduced sensitivity to cisplatin in vivo [33]. Moreover, ubenimex enhanced the effect of anticancer drugs, including cisplatin, by inducing cancer cell apoptosis [34]. In our study, MT95-4 in combination with cisplatin, but not MT95-4 alone, significantly increased the number of apoptotic cells, indicating that the tumour-suppressing effect of the combination treatment might be explained by the induction of apoptosis mediated by MT95-4 by inhibiting APN/CD13 activity in tumour cells. These results indicate that MT95-4 treatment in combination with cisplatin could represent a promising therapeutic strategy for the treatment of MPM-specific tumours exhibiting high APN/CD13 expression.

There were some limitations in this study. First, the number of patients included in the prognostic analysis was small, and the significance of APN/CD13 expression in MPM needs to be validated in larger and prospective clinical studies. Second, all MPM patients in this study had undergone pre-operative chemotherapy and little is known about whether chemotherapy affects the levels of APN/CD13 expression in tumour cells. Third, we were unable to reveal the precise mechanisms underlying the antitumour and antiangiogenic effects associated with MT95-4 treatment. Therefore, further investigations to clarify this point are necessary.

In conclusion, we showed that APN/CD13 expression was potentially associated with poor prognosis in MPM patients. Moreover, our results revealed that MT95-4, a fully humanised anti-APN/CD13 mAb, suppressed tumour progression likely by inhibiting angiogenesis in an orthotopic implantation mouse model of MPM exhibiting high levels of APN/CD13 expression. Additionally, we demonstrated that MT95-4 treatment enhanced the antitumour effect associated with cisplatin, a fundamental cytotoxic agent used for MPM treatment. These findings suggest that APN/CD13 represents a potential therapeutic target in MPM and that MT95-4 in combination with cisplatin could be a promising therapeutic strategy for the treatment of MPM-specific tumours exhibiting high levels of APN/CD13 expression.

Author contributions: T. Otsuki: conception and design, collection of data, data analysis and interpretation, and manuscript writing. T. Nakashima: conception and design, data analysis and interpretation, and revision of manuscript. Y. Takayama, S. Akita: conception and design, and data analysis and interpretation. H. Hamada, T. Masuda, S. Miyamoto, Y. Horimasu, H. Iwamoto, K. Fujitaka, M. Miyake and N. Kohno: data analysis and interpretation. Y. Miyata and M. Okada: collection of data and provision of study material. N. Hattori: conception and design, financial support, data analysis and interpretation, manuscript writing, and final approval of manuscript.

Conflict of interest: None declared.

Support statement: This study was partially supported by a grant from the Ryokufukai.

\section{References}

1 Delgermaa V, Takahashi K, Park E-K, et al. Global mesothelioma deaths reported to the World Health Organization between 1994 and 2008. Bull World Health Organ 2011; 89: 716-724.

2 Murayama T, Takahashi K, Natori $\mathrm{Y}$, et al. Estimation of future mortality from pleural malignant mesothelioma in Japan based on an age-cohort model. Am J Ind Med 2006; 49: 1-7.

3 Folkman J. What is the evidence that tumors are angiogenesis dependent? J Natl Cancer Inst 1990; 82: 4-6.

4 Hlatky L, Hahnfeldt P, Folkman J. Clinical application of antiangiogenic therapy: microvessel density, what it does and doesn't tell us. J Natl Cancer Inst 2002; 94: 883-893.

5 Kumar-Singh S, Vermeulen PB, Weyler J, et al. Evaluation of tumour angiogenesis as a prognostic marker in malignant mesothelioma. J Pathol 1997; 182: 211-216. 
6 Edwards JG, Cox G, Andi A, et al. Angiogenesis is an independent prognostic factor in malignant mesothelioma. Br J Cancer 2001; 85: 863-868.

7 Zalcman G, Mazieres J, Margery J, et al. Bevacizumab for newly diagnosed pleural mesothelioma in the Mesothelioma Avastin Cisplatin Pemetrexed Study (MAPS): a randomised, controlled, open-label, phase 3 trial. Lancet 2016; 387: 1405-1414.

8 Look AT, Ashmun RA, Shapiro LH, et al. Human myeloid plasma membrane glycoprotein CD13 (gp150) is identical to aminopeptidase N. J Clin Invest 1989; 83: 1299-1307.

9 Taylor A. Aminopeptidases: structure and function. FASEB J 1993; 7: 290-298.

10 Shipp MA, Look AT. Hematopoietic differentiation antigens that are membrane-associated enzymes: cutting is the key! Blood 1993; 82: 1052-1070.

11 Dixon J, Kaklamanis L, Turley H, et al. Expression of aminopeptidase-N (CD 13) in normal tissues and malignant neoplasms of epithelial and lymphoid origin. J Clin Pathol 1994; 47: 43-47.

12 Favaloro EJ. CD-13 ('gp150'; aminopeptidase-N): co-expression on endothelial and haemopoietic cells with conservation of functional activity. Immunol Cell Biol 1991; 69: 253-260.

13 Saiki I, Fujii H, Yoneda J, et al. Role of aminopeptidase N (CD13) in tumor-cell invasion and extracellular matrix degradation. Int J Cancer 1993; 54: 137-143.

14 Yoneda J, Saiki I, Fujii H, et al. Inhibition of tumor invasion and extracellular matrix degradation by ubenimex (bestatin). Clin Exp Metastasis 1992; 10: 49-59.

15 Fujii H, Nakajima M, Saiki I, et al. Human melanoma invasion and metastasis enhancement by high expression of aminopeptidase N/CD13. Clin Exp Metastasis 1995; 13: 337-344.

16 Bhagwat SV, Lahdenranta J, Giordano R, et al. CD13/APN is activated by angiogenic signals and is essential for capillary tube formation. Blood 2001; 97: 652-659.

17 Pasqualini R, Koivunen E, Kain R, et al. Aminopeptidase $\mathrm{N}$ is a receptor for tumor-homing peptides and a target for inhibiting angiogenesis. Cancer Res 2000; 60: 722-727.

18 Fukasawa K, Fujii H, Saitoh Y, et al. Aminopeptidase N (APN/CD13) is selectively expressed in vascular endothelial cells and plays multiple roles in angiogenesis. Cancer Lett 2006; 243: 135-143.

19 Hashida H, Takabayashi A, Kanai M, et al. Aminopeptidase $\mathrm{N}$ is involved in cell motility and angiogenesis: its clinical significance in human colon cancer. Gastroenterology 2002; 122: 376-386.

20 Ikeda N, Nakajima Y, Tokuhara T, et al. Clinical significance of aminopeptidase N/CD13 expression in human pancreatic carcinoma. Clin Cancer Res 2003; 9: 1503-1508.

21 Tokuhara $\mathrm{T}$, Hattori N, Ishida $\mathrm{H}$, et al. Clinical significance of aminopeptidase $\mathrm{N}$ in non-small cell lung cancer. Clin Cancer Res 2006; 12: 3971-3978.

22 Dalal BI, Al Mugairi A, Pi S, et al. Aberrant expression of CD13 identifies a subgroup of standard-risk adult acute lymphoblastic leukemia with inferior survival. Clin Lymphoma Myeloma Leuk 2014; 14: 239-244.

23 Nakataki E, Yano S, Matsumori Y, et al. Novel orthotopic implantation model of human malignant pleural mesothelioma (EHMES-10 cells) highly expressing vascular endothelial growth factor and its receptor. Cancer Sci 2006; 97: 183-191.

24 Yokoyama A, Kohno N, Fujino S, et al. Origin of heterogeneity of interleukin-6 (IL-6) levels in malignant pleural effusions. Oncol Rep 1994; 1: 507-511.

25 Akita S, Hattori N, Masuda T, et al. MT95-4, a fully humanized antibody raised against aminopeptidase N, reduces tumor progression in a mouse model. Cancer Sci 2015; 106: 921-928.

26 Takayama Y, Hattori N, Hamada H, et al. Inhibition of PAI-1 limits tumor angiogenesis regardless of angiogenic stimuli in malignant pleural mesothelioma. Cancer Res 2016; 76: 3285-3294.

27 Guzman-Rojas L, Rangel R, Salameh A, et al. Cooperative effects of aminopeptidase N (CD13) expressed by nonmalignant and cancer cells within the tumor microenvironment. Proc Natl Acad Sci USA 2012; 109: $1637-1642$.

28 Menrad A, Speicher D, Wacker J, et al. Biochemical and functional characterization of aminopeptidase $\mathrm{N}$ expressed by human melanoma cells. Cancer Res 1993; 53: 1450-1455.

29 Moses MA. The regulation of neovascularization of matrix metalloproteinases and their inhibitors. Stem Cells 1997; 15: 180-189.

30 Bauvois B. Transmembrane proteases in cell growth and invasion: new contributors to angiogenesis? Oncogene 2004; 23: 317-329.

31 Rangel R, Sun Y, Guzman-Rojas L, et al. Impaired angiogenesis in aminopeptidase N-null mice. Proc Natl Acad Sci USA 2007; 104: 4588-4593.

32 Ichinose $\mathrm{Y}$, Genka K, Koike T, et al. Randomized double-blind placebo-controlled trial of bestatin in patients with resected stage I squamous-cell lung carcinoma. J Natl Cancer Inst 2003; 95: 605-610.

33 van Hensbergen Y, Broxterman HJ, Rana S, et al. Reduced growth, increased vascular area, and reduced response to cisplatin in CD13-overexpressing human ovarian cancer xenografts. Clin Cancer Res 2004; 10: 1180-1191.

34 Yamashita $\mathrm{M}$, Wada $\mathrm{H}$, Eguchi $\mathrm{H}$, et al. A CD13 inhibitor, ubenimex, synergistically enhances the effects of anticancer drugs in hepatocellular carcinoma. Int J Oncol 2016; 49: 89-98. 